\title{
Gender Bias in Utilization of Health Services in Chandigarh
}

Sir,

Male-female differentials in mortality patterns among children in developing countries have been well documented, many countries showing, how any biological advantage of females is overridden by societal norms so that female mortality far exceeds male mortality after perinatal and neonatal period. ${ }^{1,2}$ As per the National Family Health Survey (NFHS) III, infant mortality, child mortality and under-five mortality, all three rates exceed in females as compared to males in India. ${ }^{3}$ This difference in mortality is the clearest sign of overt and covert discriminatory behavioral practices which favor the treatment of sons over daughter. The NFHS III also reveals a continued son preference over daughter by both men and women, especially in the northern part of the country. ${ }^{3}$ Discrimination against girls in India, especially in the northern region is well documented. ${ }^{4}$ Chandigarh is a union territory with a low female: male ratio (777 females per 1000 males), and has a high literacy rate of 81.9 as compared to 64.8 of national. ${ }^{5}$ The present study investigates the extent of sex bias in utilization of health care facilities for children in our hospital.

Data were obtained on all outpatients and admissions from January 2005 to December 2006 in the Department of Pediatrics, Government Medical College \& Hospital, Chandigarh. We studied gender related data on following parameters: 1. New OPD patients; 2. Revisit of patients in OPD; 3 . Indoor admissions; 4. Utilization of UIP vaccines; 5 . Utilization of optional vaccines; 6. Babies left against medical advice (LAMA) from neonatal nursery. Rates among males and females were compared using normal tests of proportions after adjusting differentials in birth rates. The results are presented in Table 1.

TABLE 1. Birth Rate Adjusted Distribution of Children in Relation to Sex

\begin{tabular}{llll}
\hline Parameters & Male & Female & p value \\
\hline New OPD patients & $4470(54.60)$ & $3713(45.40)$ & 0.001 \\
OPD Revisits & $29332(57.00)$ & $22163(43.00)$ & 0.001 \\
Indoor admissions & $4581(55.80)$ & $3633(44.20)$ & 0.001 \\
UIP vaccines & $18615(51.80)$ & $17333(48.20)$ & 0.001 \\
Optional vaccines & $6735(50.9)$ & $6475(49.1)$ & 0.02 \\
LAMA & & $39(66.1)$ & 0.05 \\
(Neonatal nursery) & $20(33.9)$ & &
\end{tabular}

Number in parenthesis indicate percentages
We found that all six parameters studied were significantly unfavorable to female children pointing that, there exists a bias in the utilization of health care. Several studies in Punjab have shown differences in health care utilization for girls, medical attention being sought much less frequently and only at a much later stage of illness. ${ }^{4,6}$ Lower socioeconomic status, higher number of female siblings, and an increasing distance of health facility from home, have been associated with greater discrimination against girls. ${ }^{7}$ In contrast, the per-capita income and literacy rate in Chandigarh are much higher than national average, still gender bias has been observed in our hospital. Moreover, we observed gender bias in the utilization of UIP vaccines, which are administered free of cost in our hospital, negating that economic status could be a reason of decreased utilization of vaccines for female child. A detailed analysis of reasons other than above for such a sex bias in health care utilization in Chandigarh is needed.

Abhijeet Saha, Veena Parmar, Deepak Chawala
and Dinesh Walia' [DOI-10.1007/s12098-009-0095-0]

\section{REFERENCES}

1. Lopez AD. Sex differentials in mortality. WHO chronicle $1984 ; 38: 217-224$

2. D'Souza S, Chen L. Sex differentials in mortality in rural Bangladesh. Popul Dev Rev 1980; 6: 257-270

3. National Family Health Survey-3, Ministry of Health and Family Welfare, Government of India; International Institute of Population Sciences 2005-06.

4. Booth BE, Verma M. Decreased access to medical care for girls in Punjab, India. The roles of age, religion and distance. Am J Public Health 1992; 82:1155-1157

5. Census of India. Office of registrar general and census commissioner, India. Available from: URL: http:// www.censusindia.gov.in/.Accessed February 7, 2008.

6. Singh S. Medical care in fatal illness of a rural Punjab population: some social, biological and cultural factors and their ecological implications. Indian J Med Research 1962; 50: 865-880

7. Gupta MD. Selective discrimination against female children in rural Punjab, India. Popul Dev Rev 1987; 13:77-100 